\title{
AN EXISTENCE RESULT OF ONE NONTRIVIAL SOLUTION FOR TWO POINT BOUNDARY VALUE PROBLEMS
}

\author{
GABRIELE BONANNO ${ }^{\bowtie}$ and ANGELA SCIAMMETTA
}

(Received 28 November 2010)

\begin{abstract}
Existence results of positive solutions for a two point boundary value problem are established. No asymptotic condition on the nonlinear term either at zero or at infinity is required. A classical result of Erbe and Wang is improved. The approach is based on variational methods.
\end{abstract}

2010 Mathematics subject classification: primary 34B15.

Keywords and phrases: boundary value problem, positive solutions.

\section{Introduction}

The aim of this paper is to establish existence results of nontrivial solutions for the ordinary Dirichlet boundary value problem

$$
\left\{\begin{array}{l}
-u^{\prime \prime}=\lambda f(x, u) \quad \text { for } x \in(0,1), \\
u(0)=u(1)=0
\end{array}\right.
$$

where $f:[0,1] \times \mathbb{R} \rightarrow \mathbb{R}$ is an $L^{1}$-Carathéodory function and $\lambda$ is a positive real parameter. The existence of solutions for problem $\left(D_{\lambda}\right)$ and, in particular, for problem $\left(D_{1}\right)$ or, more generally, for nonlinear differential problems has been widely investigated (see, for instance, [1, 2, 6, 8-12, 14] and the references therein). In particular, when $f(x, u)=\alpha(x) g(u)$, in the seminal work [9] the following asymptotic conditions:

$$
\lim _{u \rightarrow 0} \frac{g(u)}{u}=\infty \quad \text { and } \quad \lim _{u \rightarrow \infty} \frac{g(u)}{u}=0
$$

(or, alternatively, $\lim _{u \rightarrow 0}(g(u) / u)=0$ and $\lim _{u \rightarrow \infty}(g(u) / u)=\infty$ ) are required. Also, in $[6,8,10-12,14]$ asymptotic conditions on the nonlinear term are assumed.

In our main result (Theorem 3.1), as well as in its consequences (Corollary 3.2 and Theorem 3.3), no asymptotic condition on the nonlinear term is assumed. Moreover,

(C) 2011 Australian Mathematical Publishing Association Inc. 0004-9727/2011 \$16.00 
in a further consequence of Theorem 3.1 (that is, Theorem 3.8) only the condition $\lim _{u \rightarrow 0^{+}}(g(u) / u)=\infty$ is enough to ensure the existence of parameters $\lambda$ for which the problem $\left(D_{\lambda}\right)$ admits one positive solution, without assuming further conditions on $g$, contrary to results in [6, 8-10,14]. In particular, simple examples show that our theorems and results in $[8,10,14]$ are mutually independent (see Remark 3.11 and Example 3.10) and that Theorem 3.3 improves [9, Theorem 1(ii)] (see Remark 3.5).

In our results, instead of asymptotic conditions on the nonlinear term, a suitable algebraic inequality which involves the potential of the nonlinear term is required (see condition (3.2) in Theorem 3.3). As an example, we state here a special case.

THEOREM 1.1. Let $g: \mathbb{R} \rightarrow \mathbb{R}$ be a nonnegative continuous function such that

$$
\int_{0}^{4} g(t) d t<4 \int_{0}^{1} g(t) d t .
$$

Then for each $\lambda \in\left(16 / \int_{0}^{1} g(t) d t, 64 / \int_{0}^{4} g(t) d t\right)$ the problem

$$
\left\{\begin{array}{l}
-u^{\prime \prime}=\lambda \times g(u) \\
u(0)=u(1)=0
\end{array} \quad \text { for } x \in(0,1),\right.
$$

admits one positive classical solution $u$ such that

$$
|u(x)|<4 \text { for all } x \in[0,1] .
$$

Our main tool is a critical point theorem (see Theorem 2.1), which is a more precise version of the Ricceri variational principle [13, Theorem 2.5] and of [5, Theorem 3.1].

This note is arranged as follows. In Section 2, we recall some basic definitions, while Section 3 is devoted to existence results for problem $\left(D_{\lambda}\right)$.

\section{Preliminaries and basic notation}

Our main tool is the Ricceri variational principle [13, Theorem 2.5] as given below (see Remark 2.2). First, given $\Phi, \Psi: X \rightarrow \mathbb{R}$, put

$$
\beta\left(r_{1}, r_{2}\right)=\inf _{v \in \Phi^{-1}\left(\left(r_{1}, r_{2}\right)\right)} \frac{\sup _{u \in \Phi^{-1}\left(\left(r_{1}, r_{2}\right)\right)} \Psi(u)-\Psi(v)}{r_{2}-\Phi(v)}
$$

and

$$
\rho_{2}\left(r_{1}, r_{2}\right)=\sup _{v \in \Phi^{-1}\left(\left(r_{1}, r_{2}\right)\right)} \frac{\Psi(v)-\sup _{u \in \Phi^{-1}\left(\left(-\infty, r_{1}\right]\right)} \Psi(u)}{\Phi(v)-r_{1}}
$$

for all $r_{1}, r_{2} \in \mathbb{R}$, with $r_{1}<r_{2}$.

THEOREM 2.1. Let $X$ be a reflexive real Banach space; $\Phi: X \rightarrow \mathbb{R}$ be a sequentially weakly lower semicontinuous, coercive, and continuously Gâteaux differentiable function; $\Psi: X \rightarrow \mathbb{R}$ be a sequentially weakly upper semicontinuous and continuously 
Gâteaux differentiable function. Put $I_{\lambda}=\Phi-\lambda \Psi$ and assume that there are $r_{1}$, $r_{2} \in \mathbb{R}$, with $r_{1}<r_{2}$, such that

$$
\beta\left(r_{1}, r_{2}\right)<\rho_{2}\left(r_{1}, r_{2}\right),
$$

where $\beta$ and $\rho_{2}$ are given by (2.1) and (2.2).

Then for each $\lambda \in\left(1 / \rho_{2}\left(r_{1}, r_{2}\right), 1 / \beta\left(r_{1}, r_{2}\right)\right)$ there is $u_{0, \lambda} \in \Phi^{-1}\left(\left(r_{1}, r_{2}\right)\right)$ such that $I_{\lambda}\left(u_{0, \lambda}\right) \leq I_{\lambda}(u)$ for all $u \in \Phi^{-1}\left(\left(r_{1}, r_{2}\right)\right)$ and $I_{\lambda}^{\prime}\left(u_{0, \lambda}\right)=0$.

PRoOF. Fix $\lambda \in\left(1 / \rho_{2}\left(r_{1}, r_{2}\right), 1 / \beta\left(r_{1}, r_{2}\right)\right)$. Then there exist $v_{1}, v_{2} \in \Phi^{-1}\left(\left(r_{1}, r_{2}\right)\right)$ such that

$$
\frac{\sup _{u \in \Phi^{-1}\left(\left(r_{1}, r_{2}\right)\right)} \Psi(u)-\Psi\left(v_{1}\right)}{r_{2}-\Phi\left(v_{1}\right)}<\frac{1}{\lambda}<\frac{\Psi\left(v_{2}\right)-\sup _{u \in \Phi^{-1}\left(\left(-\infty, r_{1}\right]\right)} \Psi(u)}{\Phi\left(v_{2}\right)-r_{1}} .
$$

Hence, by putting

$$
\Phi\left(v_{0}\right)-\lambda \Psi\left(v_{0}\right)=\min \left\{\Phi\left(v_{1}\right)-\lambda \Psi\left(v_{1}\right), \Phi\left(v_{2}\right)-\lambda \Psi\left(v_{2}\right)\right\}
$$

one has

$$
\Phi\left(v_{0}\right)-\lambda \Psi\left(v_{0}\right)<r_{1}-\sup _{u \in \Phi^{-1}\left(\left(-\infty, r_{1}\right]\right)} \lambda \Psi(u)
$$

and

$$
\Phi\left(v_{0}\right)-\lambda \Psi\left(v_{0}\right)<r_{2}-\sup _{u \in \Phi^{-1}\left(\left(r_{1}, r_{2}\right)\right)} \lambda \Psi(u)
$$

Now, put

$$
\begin{array}{r}
M=r_{2}-\Phi\left(v_{0}\right)+\lambda \Psi\left(v_{0}\right), \\
(\lambda \Psi)_{M}(u)= \begin{cases}\lambda \Psi(u) & \text { if } \lambda \Psi(u)<M, \\
M & \text { if } \lambda \Psi(u) \geq M,\end{cases} \\
\Phi^{r_{1}}(u)= \begin{cases}\Phi(u) & \text { if } \Phi(u)>r_{1}, \\
r_{1} & \text { if } \Phi(u) \leq r_{1},\end{cases}
\end{array}
$$

and

$$
J=\Phi^{r_{1}}-(\lambda \Psi)_{M}
$$

Clearly, $J$ is sequentially weakly lower semicontinuous. Moreover, since $\Phi$ is coercive, $J$ is also coercive. From the classical theorem of the direct methods (see, for instance, [15, Theorem 1.2]), it follows that $J$ admits a global minimum in $X$, that is, there is $u_{0} \in X$ such that

$$
J\left(u_{0}\right) \leq J(u) \text { for all } u \in X .
$$

Now, we claim that there is $u_{0, \lambda} \in \Phi^{-1}\left(\left(r_{1}, r_{2}\right)\right)$ such that

$$
J\left(u_{0, \lambda}\right) \leq J(u) \text { for all } u \in X .
$$


First, we observe that from (2.6) one has $J\left(u_{0}\right) \leq J\left(v_{0}\right)$. Therefore, if $J\left(u_{0}\right)=$ $J\left(v_{0}\right)$, by choosing $u_{0, \lambda}=v_{0},(2.7)$ holds. So we can assume that

$$
J\left(u_{0}\right)<J\left(v_{0}\right)
$$

and our aim is then to show that $u_{0} \in \Phi^{-1}\left(\left(r_{1}, r_{2}\right)\right)$. To this end, we prove first that $\Phi\left(u_{0}\right)>r_{1}$. Suppose on the contrary that $\Phi\left(u_{0}\right) \leq r_{1}$. From (2.4), we have

$$
\lambda \Psi\left(u_{0}\right)<r_{1}-\Phi\left(v_{0}\right)+\lambda \Psi\left(v_{0}\right)<r_{2}-\Phi\left(v_{0}\right)+\lambda \Psi\left(v_{0}\right)=M,
$$

that is,

$$
\lambda \Psi\left(u_{0}\right)<M .
$$

Moreover, again from (2.4), taking (2.9) into account, one has

$$
J\left(v_{0}\right)=\Phi\left(v_{0}\right)-\lambda \Psi\left(v_{0}\right)<r_{1}-\lambda \Psi\left(u_{0}\right)=\Phi^{r_{1}}\left(u_{0}\right)-(\lambda \Psi)_{M}\left(u_{0}\right)=J\left(u_{0}\right),
$$

which contradicts (2.6). Hence, $\Phi\left(u_{0}\right)>r_{1}$ is proved.

Now, we show that $\Phi\left(u_{0}\right)<r_{2}$. From (2.8), one has

$$
\Phi^{r_{1}}\left(u_{0}\right)-(\lambda \Psi)_{M}\left(u_{0}\right)<\Phi\left(v_{0}\right)-\lambda \Psi\left(v_{0}\right)
$$

and

$$
\Phi\left(u_{0}\right)<(\lambda \Psi)_{M}\left(u_{0}\right)+\Phi\left(v_{0}\right)-\lambda \Psi\left(v_{0}\right) \leq M+\Phi\left(v_{0}\right)-\lambda \Psi\left(v_{0}\right)=r_{2},
$$

that is, $\Phi\left(u_{0}\right)<r_{2}$. Hence, (2.7) holds.

Finally, we show that $u_{0, \lambda}$ satisfies our conclusion. To this end, we observe that for all $u \in \Phi^{-1}\left(\left(r_{1}, r_{2}\right)\right)$, from (2.5) one has $\lambda \Psi(u)<r_{2}-\Phi\left(v_{0}\right)+\lambda \Psi\left(v_{0}\right)=M$. Therefore (2.7) becomes

$$
\Phi\left(u_{0, \lambda}\right)-\lambda \Psi\left(u_{0, \lambda}\right) \leq \Phi(u)-\lambda \Psi(u)
$$

for all $u \in \Phi^{-1}\left(\left(r_{1}, r_{2}\right)\right)$ and the conclusion is achieved.

REMARK 2.2. Theorem 2.1, as well as [5, Theorem 3.1], improves [13, Theorem 2.5], since the weak closure of the sublevels of $\Phi$ is not used (see [5, Remark 3.3]). We also observe that, by choosing $r_{1}<\inf _{X} \Phi$, Theorem 2.1 gives back again the Ricceri variational principle [13, Theorem 2.5] and [5, Remark 3.3], while Theorems 3.1 and 3.3 below are examples where Theorem 2.1 is applied, by choosing $r_{1} \geq \inf _{X} \Phi$.

REMARK 2.3. If, in addition, we assume that the Gâteaux derivative of $\Phi$ admits a continuous inverse on $X^{*}$ and the Gâteaux derivative of $\Psi$ is compact, Theorem 2.1 can be deduced from [4, Theorem 5.1], taking [4, Proposition 2.1] into account. On the other hand, [4, Theorem 5.1], under suitable hypotheses, ensures the same conclusion of Theorem 2.1 without requiring any weak continuity assumption on $\Phi$ and $\Psi$. 
Let $X$ be the Sobolev space $W_{0}^{1,2}([0,1])$ endowed with the norm

$$
\|u\|:=\left(\int_{0}^{1}\left|u^{\prime}(x)\right|^{2} d x\right)^{\frac{1}{2}} .
$$

Throughout, $f:[0,1] \times \mathbb{R} \rightarrow \mathbb{R}$ is an $L^{1}$-Carathéodory function and $\lambda$ is a positive real parameter. We recall that $f:[0,1] \times \mathbb{R} \rightarrow \mathbb{R}$ is an $L^{1}$-Carathéodory function if:

(a) $x \mapsto f(x, \xi)$ is measurable for every $\xi \in \mathbb{R}$;

(b) $\xi \mapsto f(x, \xi)$ is continuous for almost every $x \in[0,1]$;

(c) for every $s>0$ there exists a function $l_{s} \in L^{1}([0,1])$ such that

$$
\sup _{|\xi| \leq s}|f(x, \xi)| \leq l_{s}(x)
$$

$|\xi| \leq s$

for almost every $x \in[0,1]$.

Put

$$
F(x, t)=\int_{0}^{t} f(x, \xi) d \xi \quad \text { for all }(x, t) \in[0,1] \times \mathbb{R} .
$$

We recall that $u:[0,1] \rightarrow \mathbb{R}$ is a weak solution of problem $\left(D_{\lambda}\right)$ if $u \in W_{0}^{1,2}([0,1])$ satisfies the following condition:

$$
\int_{0}^{1} u^{\prime}(x) v^{\prime}(x) d x=\lambda \int_{0}^{1} f(x, u(x)) v(x) d x \quad \text { for all } v \in W_{0}^{1,2}([0,1]) .
$$

Clearly, when $f$ is continuous in $[0,1] \times \mathbb{R}$ the weak solutions for $\left(D_{\lambda}\right)$ are classical solutions.

Finally, we recall the following inequality, which we will use later:

$$
|u(x)|<\frac{1}{2}\|u\| \quad \text { for all } x \in[0,1] \text { and for all } u \in X .
$$

\section{Main results}

In this section we present our main results.

Given three nonnegative constants $c_{1}, c_{2}$, $d$, with $c_{1}<\sqrt{2} d<c_{2}$, put

$$
\begin{aligned}
& a\left(c_{2}, d\right):= \\
& \quad \frac{\int_{0}^{1} \max _{|\xi| \leq c_{2}} F(x, \xi) d x-\left[\int_{\frac{1}{4}}^{\frac{3}{4}} F(x, d) d x+\frac{1}{4 d} \int_{0}^{d} F\left(\frac{x}{4 d}, x\right) d x+\frac{1}{4 d} \int_{0}^{d} F\left(1-\frac{x}{4 d}, x\right) d x\right]}{2 c_{2}^{2}-4 d^{2}}
\end{aligned}
$$

and

$$
\begin{aligned}
& b\left(c_{1}, d\right):= \\
& \quad \frac{\left[\int_{\frac{1}{4}}^{\frac{3}{4}} F(x, d) d x+\frac{1}{4 d} \int_{0}^{d} F\left(\frac{x}{4 d}, x\right) d x+\frac{1}{4 d} \int_{0}^{d} F\left(1-\frac{x}{4 d}, x\right) d x\right]-\int_{0}^{1} \max _{|\xi| \leq c_{1}} F(x, \xi) d x}{4 d^{2}-2 c_{1}^{2}} .
\end{aligned}
$$


THEOREM 3.1. Assume that there exist three constants $c_{1}, c_{2}, d$, with $0 \leq c_{1}<$ $\sqrt{2} d<c_{2}$, such that

$$
a\left(c_{2}, d\right)<b\left(c_{1}, d\right) .
$$

Then, for each $\lambda \in\left(1 / b\left(c_{1}, d\right), 1 / a\left(c_{2}, d\right)\right)$, the problem $\left(D_{\lambda}\right)$ admits at least one nontrivial weak solution $\bar{u}$ such that $2 c_{1}<\|\bar{u}\|<2 c_{2}$.

PROOF. Put

$$
\Phi(u):=\frac{1}{2}\|u\|^{2} \quad \text { and } \quad \Psi(u):=\int_{0}^{1} F(x, u(x)) d x
$$

for all $u \in X$.

It is well known that $\Phi$ and $\Psi$ satisfy all regularity assumptions requested in Theorem 2.1 and that the critical points in $X$ of the functional $\Phi-\lambda \Psi$ are precisely the weak solutions of problem $\left(D_{\lambda}\right)$. So our aim is to verify condition (2.3) of Theorem 2.1.

Now, define

$$
u_{0}(x)= \begin{cases}4 d x & \text { if } x \in\left[0, \frac{1}{4}\right), \\ d & \text { if } x \in\left[\frac{1}{4}, \frac{3}{4}\right], \\ 4 d(1-x) & \text { if } x \in\left(\frac{3}{4}, 1\right],\end{cases}
$$

and put $r_{1}=2 c_{1}^{2}$ and $r_{2}=2 c_{2}^{2}$.

Clearly, $u_{0} \in W_{0}^{1,2}([0,1])$,

$$
\Psi\left(u_{0}\right)=\int_{\frac{1}{4}}^{\frac{3}{4}} F(x, d) d x+\frac{1}{4 d} \int_{0}^{d} F\left(\frac{x}{4 d}, x\right) d x+\frac{1}{4 d} \int_{0}^{d} F\left(1-\frac{x}{4 d}, x\right) d x
$$

and

$$
\Phi\left(u_{0}\right)=4 d^{2} .
$$

Since $c_{1}<\sqrt{2} d<c_{2}$, one has $r_{1}<\Phi\left(u_{0}\right)<r_{2}$.

Moreover, for all $u \in X$ such that $u \in \Phi^{-1}\left(\left(-\infty, r_{2}\right)\right)$ one has $\|u\| \leq 2 c_{2}$ and, taking (2.10) into account, one has

$$
|u(x)|<\frac{1}{2}\|u\| \leq c_{2} \quad \text { for all } x \in[0,1] .
$$

So

$$
\Psi(u)=\int_{0}^{1} F(x, u(x)) d x \leq \int_{0}^{1} \max _{|\xi| \leq c_{2}} F(x, \xi) d x
$$

for all $u \in X$ such that $u \in \Phi^{-1}\left(\left(-\infty, r_{2}\right)\right)$. Hence

$$
\sup _{u \in \Phi^{-1}\left(\left(-\infty, r_{2}\right)\right)} \Psi(u) \leq \int_{0}^{1} \max _{|\xi| \leq c_{2}} F(x, \xi) d x .
$$


Now, arguing as before we obtain

$$
\sup _{u \in \Phi^{-1}\left(\left(-\infty, r_{1}\right]\right)} \Psi(u) \leq \int_{0}^{1} \max _{|\xi| \leq c_{1}} F(x, \xi) d x .
$$

Therefore, one has

$$
\begin{aligned}
\beta\left(r_{1},\right. & \left.r_{2}\right) \leq \frac{\sup _{u \in \Phi^{-1}\left(\left(-\infty, r_{2}\right)\right)} \Psi(u)-\Psi\left(u_{0}\right)}{r_{2}-\Phi\left(u_{0}\right)} \\
& \leq \frac{\int_{0}^{1} \max _{|\xi| \leq c_{2}} F(x, \xi) d x-\left[\int_{\frac{1}{4}}^{\frac{3}{4}} F(x, d) d x+\frac{1}{4 d} \int_{0}^{d} F\left(\frac{x}{4 d}, x\right) d x+\frac{1}{4 d} \int_{0}^{d} F\left(1-\frac{x}{4 d}, x\right) d x\right]}{2 c_{2}^{2}-4 d^{2}} \\
& =a\left(c_{2}, d\right) .
\end{aligned}
$$

On the other hand,

$$
\begin{aligned}
\rho_{2}\left(r_{1}, r_{2}\right) \geq \frac{\Psi\left(u_{0}\right)-\sup _{u \in \Phi^{-1}\left(\left(-\infty, r_{1}\right]\right)} \Psi(u)}{\Phi\left(u_{0}\right)-r_{1}} \\
\quad \geq \frac{\left[\int_{\frac{1}{4}}^{\frac{3}{4}} F(x, d) d x+\frac{1}{4 d} \int_{0}^{d} F\left(\frac{x}{4 d}, x\right) d x+\frac{1}{4 d} \int_{0}^{d} F\left(1-\frac{x}{4 d}, x\right) d x\right]-\int_{0}^{1} \max _{|\xi| \leq c_{1}} F(x, \xi) d x}{4 d^{2}-2 c_{1}^{2}} \\
=b\left(c_{1}, d\right) .
\end{aligned}
$$

So, from our assumption it follows that

$$
\beta\left(r_{1}, r_{2}\right)<\rho_{2}\left(r_{1}, r_{2}\right) .
$$

Hence, from Theorem 2.1 for each $\lambda \in\left(1 / b\left(c_{1}, d\right), 1 / a\left(c_{2}, d\right)\right), \Phi-\lambda \Psi$ admits at least one critical point $\bar{u}$ such that

$$
2 c_{1}<\|\bar{u}\|<2 c_{2}
$$

and our conclusion is achieved.

Now, we point out a previous result when the nonlinear term is with separable variables. To be precise, let $\alpha \in L^{1}([0,1])$ be such that $\alpha(x) \geq 0$ almost everywhere, $x \in[0,1], \alpha \neq \equiv$, and let $g: \mathbb{R} \rightarrow \mathbb{R}$ be a continuous function. Consider the following two point boundary value problem:

$$
\left\{\begin{array}{l}
-u^{\prime \prime}=\lambda \alpha(x) g(u) \quad \text { for } x \in(0,1), \\
u(0)=u(1)=0 .
\end{array}\right.
$$

Put

$$
G(t)=\int_{0}^{t} g(\xi) d \xi
$$

for all $t \in \mathbb{R}$. 
We have the following result.

COROLlary 3.2. Assume that $g$ is nonnegative and there exist three nonnegative constants $c_{1}, c_{2}, d$, with $c_{1}<\sqrt{2} d<c_{2}$, such that

$$
\frac{\|\alpha\|_{1} G\left(c_{2}\right)-\left(\int_{\frac{1}{4}}^{\frac{3}{4}} \alpha(x) d x\right) G(d)}{2 c_{2}^{2}-4 d^{2}}<\frac{\left(\int_{\frac{1}{4}}^{\frac{3}{4}} \alpha(x) d x\right) G(d)-\|\alpha\|_{1} G\left(c_{1}\right)}{4 d^{2}-2 c_{1}^{2}} .
$$

Then, for each

$$
\lambda \in\left(\frac{4 d^{2}-2 c_{1}^{2}}{\left(\int_{\frac{1}{4}}^{\frac{3}{4}} \alpha(x) d x\right) G(d)-\|\alpha\|_{1} G\left(c_{1}\right)}, \frac{2 c_{2}^{2}-4 d^{2}}{\|\alpha\|_{1} G\left(c_{2}\right)-\left(\int_{\frac{1}{4}}^{\frac{3}{4}} \alpha(x) d x\right) G(d)}\right),
$$

the problem $\left(A D_{\lambda}\right)$ admits at least one positive weak solution $\bar{u}$ such that $2 c_{1}<\|\bar{u}\|<$ $2 c_{2}$.

ProOf. Put $f(x, \xi)=\alpha(x) g(\xi)$ for all $(x, \xi) \in[0,1] \times \mathbb{R}$. Since $g$ is a nonnegative function, one has

$$
\int_{0}^{1} \max _{|\xi| \leq c_{2}} F(x, \xi) d x=\|\alpha\|_{1} G\left(c_{2}\right), \quad \int_{0}^{1} \max _{|\xi| \leq c_{1}} F(x, \xi) d x=\|\alpha\|_{1} G\left(c_{1}\right),
$$

and

$$
\frac{1}{4 d} \int_{0}^{d} F\left(\frac{x}{4 d}, x\right) d x+\frac{1}{4 d} \int_{0}^{d} F\left(1-\frac{x}{4 d}, x\right) d x \geq 0 .
$$

Therefore, one has

$$
\begin{gathered}
a\left(c_{2}, d\right) \leq \frac{\|\alpha\|_{1} G\left(c_{2}\right)-\left(\int_{\frac{1}{4}}^{\frac{3}{4}} \alpha(x) d x\right) G(d)}{2 c_{2}^{2}-4 d^{2}} \\
\text { and } \quad b\left(c_{1}, d\right) \geq \frac{\left(\int_{\frac{1}{4}}^{\frac{3}{4}} \alpha(x) d x\right) G(d)-\|\alpha\|_{1} G\left(c_{1}\right)}{4 d^{2}-2 c_{1}^{2}} .
\end{gathered}
$$

Hence, taking (3.1) into account, Theorem 3.1 and the strong maximum principle ensure the conclusion.

Now, we point out two relevant consequences of Corollary 3.2, namely Theorems 3.3 and 3.8 .

THEOREM 3.3. Assume that $g$ is nonnegative and there exist two positive constants $c$, $d$, with $\sqrt{2} d<c$, such that

$$
\frac{G(c)}{c^{2}}<\left(\frac{\int_{\frac{1}{4}}^{\frac{3}{4}} \alpha(x) d x}{2\|\alpha\|_{1}}\right) \frac{G(d)}{d^{2}} .
$$


Then, for each

$$
\lambda \in\left(\frac{4}{\int_{\frac{1}{4}}^{\frac{3}{4}} \alpha(x) d x} \frac{d^{2}}{G(d)}, \frac{2}{\|\alpha\|_{1}} \frac{c^{2}}{G(c)}\right)
$$

the problem $\left(A D_{\lambda}\right)$ admits at least one positive weak solution $\bar{u}$ such that $|\bar{u}(x)|<c$ for all $x \in[0,1]$.

PROOF. Our aim is to apply Corollary 3.2. To this end, we pick $c_{1}=0$ and $c_{2}=c$. From (3.2), one has

$$
\frac{\|\alpha\|_{1} G\left(c_{2}\right)-\left(\int_{\frac{1}{4}}^{\frac{3}{4}} \alpha(x) d x\right) G(d)}{2 c_{2}^{2}-4 d^{2}}<\frac{\|\alpha\|_{1} G\left(c_{2}\right)-2\|\alpha\|_{1} d^{2} G\left(c_{2}\right) / c_{2}^{2}}{2 c_{2}^{2}-4 d^{2}}=\frac{\|\alpha\|_{1} G(c)}{2 c^{2}} .
$$

On the other hand, one has

$$
\frac{\left(\int_{\frac{1}{4}}^{\frac{3}{4}} \alpha(x) d x\right) G(d)-\|\alpha\|_{1} G\left(c_{1}\right)}{4 d^{2}-2 c_{1}^{2}}=\frac{\left(\int_{\frac{1}{4}}^{\frac{3}{4}} \alpha(x) d x\right) G(d)}{4 d^{2}} .
$$

Hence, from Corollary 3.2, taking (2.10) into account, the conclusion follows.

REMARK 3.4. Theorem 1.1 in the Introduction is a consequence of Theorem 3.3 by choosing $\alpha(x)=x$ for all $x \in[0,1], d=1$, and $c=4$.

REMARK 3.5. In [9, Theorem 1(ii)], the conditions

$$
\lim _{t \rightarrow 0} \frac{g(t)}{t}=+\infty, \quad \lim _{t \rightarrow \infty} \frac{g(t)}{t}=0
$$

ensure the existence of at least one positive solution for $\left(A D_{1}\right)$. Clearly, conditions $(E)$ imply (3.2) and Theorem 3.3 establishes the existence of at least one positive solution to $\left(A D_{\lambda}\right)$ for all $\lambda>0$. On the other hand, simple examples of functions, which satisfy (3.2) and do not satisfy conditions $(E)$, can be found (see, for instance, Example 3.10 and Remark 3.11). Hence Theorem 3.3 improves [9, Theorem 1(ii)].

REMARK 3.6. We explicitly observe that, applying the classical Ricceri variational principle (or the same Theorem 2.1 by choosing $r_{1}<0$ ) and arguing as in the proof of Theorem 3.1, the following result can be obtained:

Given a nonnegative continuous function $g: \mathbb{R} \rightarrow \mathbb{R}$ and fixed a positive constant $c$, one has that for each $\lambda \in\left(0,\left(2 /\|\alpha\|_{1}\right)\left(c^{2} / G(c)\right)\right)$, the problem $\left(A D_{\lambda}\right)$ admits at least one nonnegative weak solution $\bar{u}$ such that $|\bar{u}(x)|<c$ for all $x \in[0,1]$.

Clearly, in this case the solution may be zero.

An immediate consequence of Theorem 3.3 and the preceding remark is the following corollary. 
COROLlary 3.7. Let $g: \mathbb{R} \rightarrow \mathbb{R}$ be a nonnegative continuous function such that $g(0) \neq 0$ and assume that there exist two positive constants $c, d$ such that

$$
\frac{G(c)}{c^{2}}<\left(\frac{\int_{\frac{1}{4}}^{\frac{3}{4}} \alpha(x) d x}{2\|\alpha\|_{1}}\right) \frac{G(d)}{d^{2}} .
$$

Then, for each

$$
\lambda \in\left(\frac{4}{\int_{\frac{1}{4}}^{\frac{3}{4}} \alpha(x) d x} \frac{d^{2}}{G(d)}, \frac{2}{\|\alpha\|_{1}} \frac{c^{2}}{G(c)}\right),
$$

the problem $\left(A D_{\lambda}\right)$ admits at least one positive classical solution $\bar{u}$ such that $|\bar{u}(x)|<$ $c$ for all $x \in[0,1]$.

Proof. From (3.3), one has $c \neq \sqrt{2} d$. If $c>\sqrt{2} d$, Theorem 3.3 ensures the conclusion, while if $c<\sqrt{2} d$ the result in Remark 3.6 completes the proof.

A further consequence of Theorem 3.1 is the following result.

THEOREM 3.8. Assume that

$$
\lim _{t \rightarrow 0^{+}} \frac{g(t)}{t}=+\infty
$$

fix $\delta>0$ such that $g(t)>0$ for all $t \in(0, \delta)$, and put $\lambda^{*}=2 /\|\alpha\|_{1} \sup _{c \in(0, \delta)} c^{2} / G(c)$. Then, for each $\lambda \in\left(0, \lambda^{*}\right)$, the problem $\left(A D_{\lambda}\right)$ admits at least one positive weak solution $\bar{u}$ such that $|\bar{u}(x)|<\delta$ for all $x \in[0,1]$.

PROOF. We observe that $\lambda^{*}>0$. In fact, from (3.4) there is $\delta>0$ such that $g(t)>0$ for all $t \in(0, \delta)$. Therefore, $c^{2} / G(c)>0$ for all $c \in(0, \delta)$. Now, put

$$
g^{*}(t)= \begin{cases}g(t) & \text { if } 0<t<\delta, \\ g(0) & \text { if } t \leq 0 \\ g(\delta) & \text { if } t \geq \delta\end{cases}
$$

and denote by $G^{*}$ its antiderivative. Clearly, $g^{*}$ is continuous and nonnegative.

Fix $\bar{\lambda} \in\left(0, \lambda^{*}\right)$. Then there is $c \in(0, \delta)$ such that $1 / \bar{\lambda}>\|\alpha\|_{1} G^{*}(c) / 2 c^{2}$. Moreover, since again from (3.4) one has

$$
\lim _{t \rightarrow 0^{+}} \frac{G^{*}(t)}{t^{2}}=+\infty
$$

there is $d>0$ such that $d<(1 / \sqrt{2}) c$ and $\left(\int_{\frac{1}{4}}^{\frac{3}{4}} \alpha(x) d x / 4\right)\left(G^{*}(d) / d^{2}\right)>1 / \bar{\lambda}$. Hence, from Theorem 3.3 the problem

$$
\left\{\begin{array}{l}
-u^{\prime \prime}=\bar{\lambda} \alpha(x) g^{*}(u) \quad \text { on }(0,1), \\
u(0)=u(1)=0
\end{array}\right.
$$


admits at least one positive solution $\bar{u}$ such that $|\bar{u}(x)|<c<\delta$ for all $x \in[0,1]$. Taking into account that $g^{*}(\bar{u}(x))=g(\bar{u}(x))$ for all $x \in[0,1]$, the conclusion is achieved.

REMARK 3.9. In particular, Theorem 3.8 ensures that if $g: \mathbb{R} \rightarrow \mathbb{R}$ is a nonnegative continuous function such that $\lim _{t \rightarrow 0^{+}}(g(t) / t)=+\infty$, then for each

$$
\lambda \in\left(0, \frac{2}{\|\alpha\|_{1}} \sup _{c \in(0,+\infty)} \frac{c^{2}}{G(c)}\right)
$$

the problem $\left(A D_{\lambda}\right)$ admits at least one positive weak solution.

EXAMPLE 3.10. Let $g_{1}: \mathbb{R} \rightarrow \mathbb{R}$ be the function defined as follows:

$$
g_{1}(t)= \begin{cases}(1+t) e^{t} & \text { if } t \leq 1 \\ h(t) & \text { if } t>1\end{cases}
$$

where $h:(1,+\infty) \rightarrow \mathbb{R}$ is a completely arbitrary function.

Owing to Theorem 3.8, for each $\lambda \in(0,4 / e)$, the following problem:

$$
\left\{\begin{array}{l}
-u^{\prime \prime}=\lambda x g_{1}(u) \quad \text { on }(0,1), \\
u(0)=u(1)=0
\end{array}\right.
$$

admits at least one positive classical solution $\bar{u}$ such that $\bar{u}(x)<1$ for all $x \in[0,1]$. In fact, it is enough to pick $\delta=1$ and to observe that

$$
\lambda^{*}=\frac{2}{\|\alpha\|_{1}} \sup _{c \in(0, \delta)} \frac{c^{2}}{\int_{0}^{c} g_{1}(t) d t}=4 \sup _{c \in(0,1)} \frac{c^{2}}{c e^{c}}=\frac{4}{e} .
$$

In particular, by choosing $\lambda=1$, the following problem:

$$
\left\{\begin{array}{l}
-u^{\prime \prime}=x(1+u) e^{u} \text { on }(0,1), \\
u(0)=u(1)=0,
\end{array}\right.
$$

admits at least one positive classical solution $\bar{u}$ such that $\bar{u}(x)<1$ for all $x \in[0,1]$.

REMARK 3.11. The existence of at least one positive solution for problem $\left(D_{\lambda}\right)$ or, more generally, for nonlinear differential problems has been widely investigated (see, for instance, [1, 3, 7] and the references therein). In particular, condition (3.4) has been assumed, for instance, in [6, 8-12]. However, in these papers, besides (3.4), further conditions must be added to obtain solutions (see [6, Theorem 3.2], [8, Lemma 3], [9, Theorem 1], [10, Lemma 3.1], [11], and [12, Corollary 1]).

In [9], together with (3.4), the condition

$$
\lim _{t \rightarrow \infty} \frac{g(t)}{t}=0
$$


is assumed. Clearly, [9, Theorem 1] cannot be applied, for instance, to problem $(P)$ of Example 3.10 since

$$
\lim _{t \rightarrow \infty} \frac{g(t)}{t}=\lim _{t \rightarrow \infty} \frac{(1+t) e^{t}}{t}=\infty .
$$

The same remark is also true for [14, Theorem 2.2], [8, Lemma 3], and [10, Lemma 3.1], where $\lim \sup _{t \rightarrow \infty}(g(t) / t)<\lambda_{1}=\pi^{2}$ is assumed.

\section{References}

[1] P. R. Agarwal, D. O'Regan and P. J. Y. Wong, Positive Solutions of Differential, Difference and Integral Equations (Kluwer, Dordrecht, 1999).

[2] D. Averna and G. Bonanno, 'A three critical points theorem and its applications to the ordinary Dirichlet problem', Topol. Methods Nonlinear Anal. 22 (2003), 93-103.

[3] C. Bereanu and J. Mawhin, 'Boundary value problems for some nonlinear systems with singular Ф-laplacian', J. Fixed Point Theory Appl. 4 (2008), 57-75.

[4] G. Bonanno, 'A critical point theorem via the Ekeland variational principle', Preprint.

[5] G. Bonanno and P. Candito, 'Non-differentiable functionals and applications to elliptic problems with discontinuous nonlinearities', J. Differential Equations 244 (2008), 3031-3059.

[6] A. Capietto and W. Dambrosio, 'Boundary value problems with sublinear conditions near zero', NoDEA: Nonlinear Differential Equations Appl. 6 (1999), 149-172.

[7] A. Capietto, J. Mawhin and F. Zanolin, 'Boundary value problems for forced superlinear second order ordinary differential equations', in: Nonlinear Partial Differential Equations, Collège de France Seminar (Longman, New York, 1994), pp. 55-94.

[8] F. Cîrstea, M. Ghergu and V. Rădulescu, 'Combined effects of asymptotically linear and singular nonlinearities in bifurcation problems of Lane-Emden-Fowler type', J. Math. Pures Appl. 84 (2005), 493-508.

[9] L. H. Erbe and H. Wang, 'On the existence of positive solutions of ordinary differential equations', Proc. Amer. Math. Soc. 120 (1994), 743-748.

[10] M. Ghergu and V. Rădulescu, 'On a class of sublinear singular elliptic problems with convection term', J. Math. Anal. Appl. 311 (2005), 635-646.

[11] N. Hirano, 'Existence of infinitely many solutions for sublinear elliptic problems', J. Math. Anal. Appl. 278 (2003), 83-92.

[12] Y. Naito and S. Tanaka, 'On the existence of multiple solutions of the boundary value problem for nonlinear second-order differential equations', Nonlinear Anal. 56 (2004), 919-935.

[13] B. Ricceri, 'A general variational principle and some of its applications', J. Comput. Appl. Math. 113 (2000), 401-410.

[14] J. Shi and M. Yao, 'Positive solutions for elliptic equations with singular nonlinearity', Electron. J. Differential Equations 4 (2005), 1-11.

[15] M. Struwe, Variational Methods (Springer, Berlin, 1996).

GABRIELE BONANNO, Department of Science for Engineering and Architecture (Mathematics Section), Engineering Faculty, University of Messina,

98166 Messina, Italy

e-mail: bonanno@unime.it

ANGELA SCIAMMETTA, Department of Mathematics, University of Messina,
98166 Messina, Italy
e-mail: asciammetta@ unime.it 